\title{
On comparison and combination of catalogues of radio source positions
}

\author{
Ju. Sokolova and Z. Malkin \\ Central Astronomical Observatory at Pulkovo RAS, Pulkovskoe Ch. 65/1, St. Petersburg 196140, Russia \\ e-mail: solei@yandex.ru
}

Received 10 March 2007 / Accepted 18 August 2007

ABSTRACT

\begin{abstract}
Context. Radio source catalogues (RSCs) obtained from very long baseline interferometry (VLBI) observations are recommended by the International Astronomical Union (IAU) as realizations of the International Celestial Reference Frame (ICRF). As the accuracy of the VLBI observations is improving with time, development of more accurate methods of RSC construction is a topical problem. Aims. In this paper we search for an optimal method of representation of the RSC systematic errors and investigate a possibility for improving the ICRF by means of combining individual RSCs.

Methods. Four methods of representing the RSC systematic differences have been examined by comparison of the differences in the radio source positions between the individual catalogues. Eight radio source catalogues and the latest ICRF realization, ICRF-Ext.2, were used for comparison. After selection of the best method of difference representation we computed the systematic differences between each individual catalogue and the ICRF. These differences were then used in the construction of the combined radio source catalogue.

Results. Representation of the systematic differences between RSCs with the Legendre-Fourier functions proved to be the most accurate method. Using this method, two combined radio source catalogues have been constructed. The first of them provides a stochastic improvement of the current realization of ICRF, and the second, final one allows us to account also for possible systematic errors in the ICRF. Comparison of the celestial pole offsets obtained from the processing of VLBI observations using ICRF and the combined catalogue has shown improvement of the results.

Conclusions. The conventional method of individual radio source catalogue comparisons used by the International Earth Rotation and Reference Systems Service does not provide sufficient accuracy and should be replaced by a more adequate method. A combined radio source catalogue allows us to improve the current realization of the ICRF.
\end{abstract}

Key words. astrometry - reference systems - techniques: interferometric - ephemerides

\section{Introduction}

The celestial reference frame (CRF), as realized by a set of coordinates for selected celestial objects, is widely used for numerous astronomy, navigation, time and other measurements. The CRF accuracy and stability are all-important for successful solutions of all these tasks. For many years, the CRF was based on optical observations and star positions. With the establishment of a new observing technique, very long baseline interferometry (VLBI), much more accurate CRF realization became available. In 1998, the CRF based on the positions of extragalactic radio sources was adopted by the IAU (International Astronomical Union) as the fundamental celestial reference frame, replacing the FK5 optical frame (Arias et al. 1995; Ma et al. 1998).

After publishing of the first VLBI RSCs, attempts were made to improve the accuracy of radio-band CRF by means of constructing combined catalogues, as it was customary for optical astronomy, where fundamental catalogues served as an international standard for astrometry and other measurements on the sky. Different methods were used to obtain a combined RSC, e.g. Walter (1989a,b), Yatskiv \& Kur'yanova (1990), Kur'yanova \& Yatskiv (1993). Also, up to 1995, IERS (International Earth Rotation Service, now International Earth Rotation and Reference Systems Service) used derived combined RSC for maintenance of the IERS Celestial Reference Frame.
However, starting from 1996, a new CRF realization was adopted by the IERS, and further approved by the IAU in 1998 . The first realization of the ICRF was based on the refined analysis of VLBI observations made at the NASA Goddard Space Flight Center, USA (Ma et al. 1998). All the 608 radio sources included in the ICRF were divided into three groups: 212 defining sources, whose coordinates are supposed to be kept in future realizations to maintain the ICRF orientation, 294 candidate sources not sufficiently monitored, and 102 other sources for improving the sky coverage. In 1999 and 2004, two ICRF extensions ICRF-Ext.1 (Ma 2001) and ICRF-Ext.2 (Fey et al. 2004) were issued. In those versions, the positions of the 212 defining sources were kept the same as those obtained in the first ICRF. It should be noted that both ICRF extensions were obtained in a manner similar to the first realization, i.e. as a result of analysis of the VLBI observations at a single analysis center. The latest ICRF realization, ICRF-Ext.2, is hereafter referred to as ICRF.

In the end of 2004, a joint pilot project of the IERS and the IVS (International VLBI Service for Geodesy and Astrometry, Schlueter et al. 2002) was initiated (Ma 2004, Call for Participation). One of the main goals of the project was to seek possible ways to improve the existing ICRF. Large experience accrued by optical astrometry over centuries shows that combining catalogues of the star positions leads to better random and systematic accuracy than individual catalogues. In 
particular, the latter can be affected by the systematic errors caused by algorithms and software used for data processing. Hopefully, a combining procedure can be used to mitigate the influence of errors in individual RSCs. Another main goal of the Pilot Project is to develop new methods of comparison of RSCs appropriate to the modern level of their precision and accuracy, i.e. their random (stochastic) and systematic errors.

The present work was performed in four steps.

1. Analysis of the random and systematic errors of individual (input) catalogues, and selection of the most adequate method of representation of the systematic differences between catalogues.

2. Determination of the systematic differences between the input catalogues and ICRF.

3. Construction of a combined catalogue in the ICRF system (stochastic improvement of the ICRF).

4. Construction of the final combined catalogue (systematic improvement of the ICRF).

First, we searched for the optimal method of representation of the RSC systematic errors. We then investigated a possibility of improving the ICRF by means of combining individual RSCs.

Four methods of analytical representation of the systematic differences between RSCs have been examined on the basis of comparison of the differences in the radio source coordinates between RSCs after applying the resultant analytical expression. Eight individual radio source catalogues and the ICRF were used in this study. After the most accurate method had been chosen, it was used to compute the systematic differences between the individual catalogues and the ICRF. Finally, these differences were used in the construction of two combined RSCs. The first combined catalogue provides a stochastic improvement of the ICRF. The final combined catalogue allows us to account also for possible systematic errors in the ICRF.

The final combined catalogue obtained was tested by means of comparison of celestial pole offset time series computed with both this RSC and the ICRF. Result of this test has shown improvement of the scatter of the time series when the final combined RSC is used.

\section{Input catalogues}

Input catalogues used in this study were submitted by eight IVS Analysis Centers: AUS (Geoscience Australia), BKG (Bundesamt für Kartographie und Geodäsie, Germany), DGFI (Deutsches Geodätisches Forschungsinstitut, Germany), GSFC (NASA Goddard Space Flight Center, USA), JPL (Caltech/NASA Jet Propulsion Laboratory, USA), MAO (Main Astronomical Observatory of National Academy of Sciences of Ukraine), SHAO (Shanghai Astronomical Observatory, China), and USNO (U.S. Naval Observatory, USA). A brief description of the input catalogues is given in Table 1 .

In this study, we took into account only the sources which have at least 15 observations in 2 sessions. After such a selection, the total number of sources present in all the input catalogues amounts to 968 .

Usually, investigation of the systematic differences between catalogues is made using a set of reference sources common for the catalogues being compared. Comparison of the lists of radio sources included in the input catalogues showed that there are 525 sources present in all the catalogues, 196 of them belonging to the list of 212 ICRF defining sources. Figure 1 shows the distribution of these two sets of the common sources over the sky.
Table 1. Input catalogues. The last column shows number of sources in the catalogue and the number of reference sources used to tie the orientation of the catalogue to the ICRF.

\begin{tabular}{llccl}
\hline \hline Center & Software & \multicolumn{2}{c}{ Time span } & \multicolumn{2}{c}{ Number of } \\
& & month/year & delays & sources \\
\hline AUS & OCCAM & $11 / 1979-12 / 2004$ & 3208197 & $737(207)$ \\
BKG & Calc/Solve & $01 / 1980-01 / 2005$ & 4031453 & $748(212)$ \\
DGFI & OCCAM & $01 / 1980-01 / 2005$ & 3650771 & $686(199)$ \\
GSFC & Calc/Solve & $08 / 1979-01 / 2005$ & 4574189 & $954(212)$ \\
JPL & MODEST & $10 / 1978-01 / 2005$ & 3575847 & $734(2)$ \\
MAO & SteelBreeze & $10 / 1980-01 / 2005$ & 3773765 & $685(25)$ \\
SHAO & Calc/Solve & $04 / 1980-01 / 2005$ & 4431503 & $813(212)$ \\
USNO & Calc/Solve & $09 / 1979-01 / 2005$ & 4252684 & $943(207)$ \\
\hline
\end{tabular}
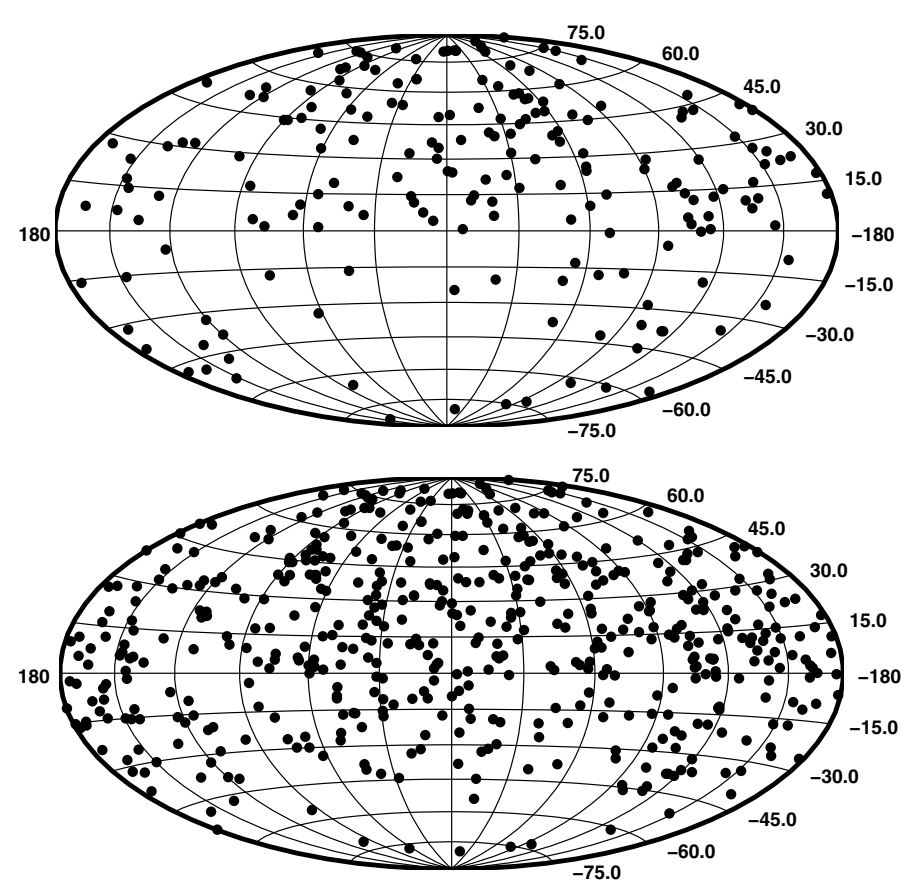

Fig. 1. Sky distribution for 196 defining sources (top) and all 525 sources (bottom) common for the input catalogues.

Both source lists, as well as other subsets amongst the 525 sources, can be used as reference for analysis of the systematic differences between catalogues. All the computations described below were carried out for both 196 and 525 sources. In this paper, we present only the results obtained with the first list of 196 sources. Although definite differences in results were found, the main conclusions made in this study do not depend on the reference source list.

Weighted root-mean-square (WRMS) differences of the radio source coordinates between the input catalogues and the ICRF for the 196 common sources are shown in Fig. 2 ( $\mu$ as stands for microarcseconds). One can see from Fig. 2 that WRMS differences have the least values for catalogues computed with Calc/Solve software, both for comparisons between catalogues, and when comparing catalogues to the ICRF. The latter is probably caused by the fact that the ICRF was constructed using Calc/Solve software. Large WRMS differences between JPL and other catalogues may be caused by its orientation to the ICRF, which has been defined by only two reference sources, unlike other catalogues, for which much longer lists of reference sources were used. Catalogue AUS shows the greatest differences with other catalogues, probably because it is the only catalogue constructed using the least squares 


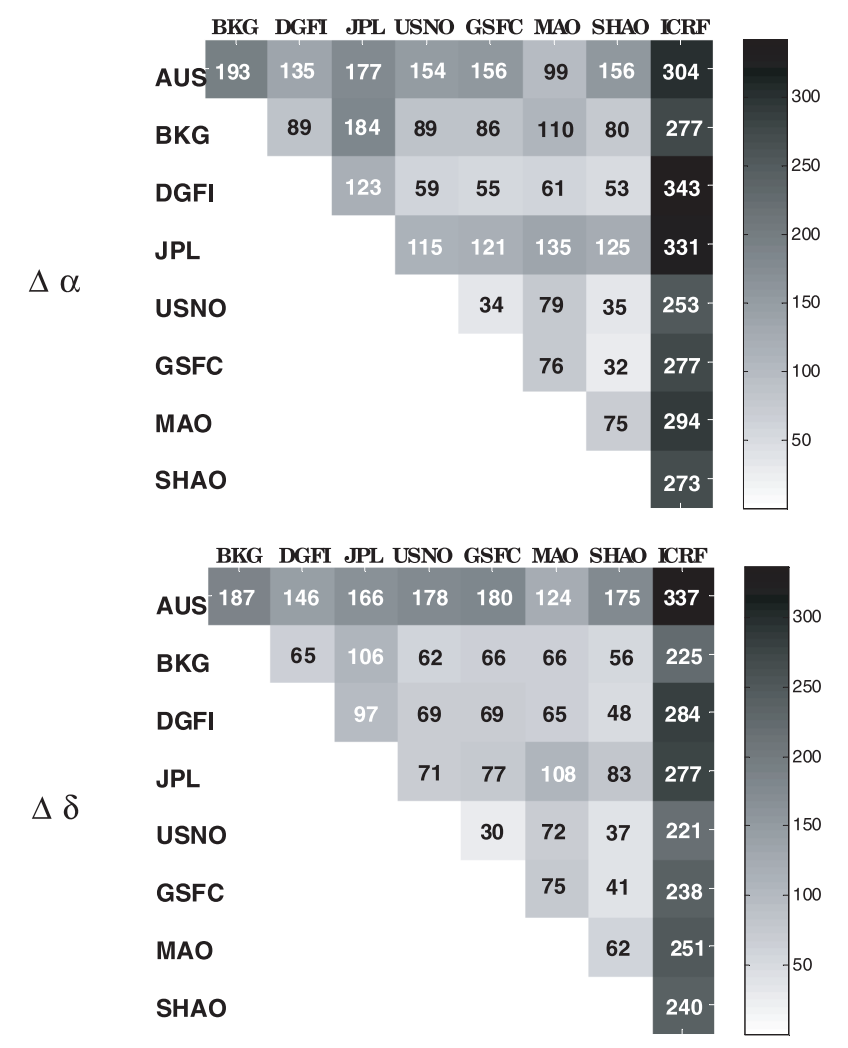

Fig. 2. WRMS differences between the input catalogues and ICRF. Unit: $\mu$ as.

collocation method, while other analysis centers used conventional least squares. One can see that the DGFI catalogue, also constructed using OCCAM software, but least squares version, does not stand out against other catalogues.

The WRMS differences between the input catalogues themselves may be not of value, especially when they are caused by the difference in orientation of the catalogues' axes, which can be easily accounted for during combination. More interestingly, all the input catalogues demonstrate rather large differences with the ICRF, which may indicate significant systematic errors in the latter. This preliminary conclusion will be confirmed by the analysis of the systematic differences given below.

\section{Analytical representation of the systematic differences}

Suppose, we have two catalogues of celestial source positions given as set of spherical coordinates $\alpha_{1 i}, \delta_{1 i}$ and $\alpha_{2 i}, \delta_{2 i}$, where $i$ is the source number. We denote the differences between spherical coordinates of the $i$ th object given in two catalogues as $\Delta \alpha_{i}=\alpha_{1 i}-\alpha_{2 i}, \Delta \delta_{i}=\delta_{1 i}-\delta_{2 i}$. The aim of an analytical representation of the systematic differences between two catalogues is to obtain formulae

$$
\begin{aligned}
& \Delta \alpha=f_{\alpha}(\alpha, \delta), \\
& \Delta \delta=f_{\delta}(\alpha, \delta),
\end{aligned}
$$$$
\text { or }
$$

$$
\left\{\begin{array}{l}
\Delta \alpha \\
\Delta \delta
\end{array}\right\}=f(\alpha, \delta),
$$

which provide minimum residuals between $\Delta \alpha_{i}, \Delta \delta_{i}$ observed and computed analytically. One can say that such a representation of the systematic differences is a kind of low-pass filter, which allow us to smooth stochastic errors in coordinates. Having such a representation, one can reduce a RSC to the system of another catalogue.

In this section, we compare four methods of analytical representation of the systematic differences between radio source positions given in various catalogues. Those methods are as follows: simple rotation around three cartesian axes hereafter referred to as "R"; rotation plus deformation used by IERS ("RD"); Brosche's method ("B"); and expansion in LegendreFourier functions ("LF").

It should be noted that in usual astrometric practice, differences between source positions in right ascension are used as $\Delta \alpha \cos \delta$, which reflects the geometry of the celestial sphere. However, hereafter we use $\Delta \alpha$ because IERS's method is formulated only for this type of difference. Other methods listed below can be easily adapted to $\Delta \alpha \cos \delta$.

\subsection{Simple rotation}

Two given catalogues realize two cartesian coordinate systems $X_{1} Y_{1} Z_{1}$ and $X_{2} Y_{2} Z_{2}$. Differences between source positions in two catalogues can be represented as the result of rotation of the second coordinate system with respect to the first coordinate system about axes $X Y Z$ by three angles $A_{1}, A_{2}, A_{3}$. The systematic differences between two catalogues can be expressed as (Walter $\&$ Sovers 2000)

$$
\begin{aligned}
\Delta \alpha & =A_{1} \tan \delta \cos \alpha+A_{2} \tan \delta \sin \alpha-A_{3}, \\
\Delta \delta & =-A_{1} \sin \alpha+A_{2} \cos \alpha .
\end{aligned}
$$

\subsection{Rotation with deformation}

This method of representation of the systematic differences between RSCs was proposed by Arias \& Bouquillon (2004), and has been used by the IERS since 1995 . The authors added to Eq. (2) three supplemental terms to account for some specific errors of VLBI catalogues. In this method, the systematic differences between two catalogues are approximated by

$$
\begin{aligned}
\Delta \alpha & =A_{1} \tan \delta \cos \alpha+A_{2} \tan \delta \sin \alpha-A_{3}+D_{\alpha} \delta, \\
\Delta \delta & =-A_{1} \sin \alpha+A_{2} \cos \alpha+D_{\delta} \delta+B_{\delta} .
\end{aligned}
$$

\subsection{Brosche's method}

In this method, as well as in the next one, an expansion of the differences in source positions between catalogues in orthogonal functions is used. Significant experience based on optical astrometry proved that such an expansion provides the highest accuracy of the representation of the systematic errors of celestial source position catalogues. In this case, the general representation of the differences (1) is given by

$$
\left\{\begin{array}{c}
\Delta \alpha \\
\Delta \delta
\end{array}\right\}=\sum_{j=0}^{g} b_{j} Y_{j}(\alpha, \delta),
$$

where $b_{j}$ are the coefficients to be found from analysis of the differences. According to Brosche (1966)

$$
Y_{j}(\alpha, \delta)= \begin{cases}P_{n 0}(\delta), & k=0, l \neq 1, \\ P_{n k}(\delta) \sin (k \alpha), & k \neq 0, l=0, \\ P_{n k}(\delta) \cos (k \alpha), & k \neq 0, l=1\end{cases}
$$


where $P_{n k}$, associated Legendre polynomials, are given by

$P_{n k}(\delta)=\cos ^{k} \delta\left[\sin ^{p} \delta+\sum_{\mu=1}^{[p / 2]} \frac{(-1)^{\mu} \prod_{v=0}^{2 \mu-1}(p-v)}{\prod_{v=1}^{\mu} 2 v(2 n-2 v+1)} \sin ^{p-2 \mu} \delta\right]$,

where $p=n-k,[p / 2]$ is the integer part of $p / 2, n=0,1, \ldots$, $k=0,1,2, \ldots, n, j=n^{2}+2 k+l-1$.

\subsection{Legendre-Fourier functions}

Bien et al. (1978) have proposed using another set of orthogonal functions for better representation of systematic differences between catalogues, especially in the polar regions. In this case (4) is given by

$$
\begin{aligned}
\left\{\begin{array}{c}
\Delta \alpha \\
\Delta \delta
\end{array}\right\} & =\sum_{n k l} b_{n k l} Y_{n k l}(\alpha, \delta), \\
Y_{n k l}(\alpha, \delta) & =R_{n k l} L_{n}(\sin \delta) F_{k l}(\alpha) .
\end{aligned}
$$

Here we omit the Hermite functions included in the original expression of Bien et al. (1978) to account for the source brightness. Generally speaking, such a dependence may exist in the case of VLBI observations too, and is worth separate investigation. Legendre polynomials can be computed using recursion:

$$
\begin{aligned}
& L_{0}=1, \\
& L_{1}=\sin \delta \\
& L_{n+1}(\sin \delta)=\frac{2 n+1}{n+1} \sin \delta L_{n}(\sin \delta)-\frac{n}{n+1} L_{n-1}(\sin \delta), \\
& \quad n=1,2, \ldots
\end{aligned}
$$

Fourier functions are given by

$F_{k l}(\alpha)= \begin{cases}1, & k=0, l=-1, \\ \cos (k l \alpha), & k \neq 0, l=1, \\ \sin (-k l \alpha), & k \neq 0, l=-1 .\end{cases}$

Lastly, the normalizing factor is

$R_{n k l}=\sqrt{2 n+1} \begin{cases}1, & k=0, \\ \sqrt{2}, & k \neq 0 .\end{cases}$

All the four methods described above were applied to the differences between each of the eight input catalogues and the ICRF for 196 common defining sources (see Sect. 2). For this purpose, the coefficients of (2), (3), (5), and (7) were found by means of least squares adjustment. We then computed the residuals between original differences and those computed by formulae (2), (3), (5), and (7). The results are presented in Fig. 3 and Table 2. Representation with Brosche's model is not shown in Fig. 3 because corresponding surfaces essentially coincide with those obtained for the expansion in the Legendre-Fourier functions. One can see that the expansion in the Legendre-Fourier functions provides the least residuals, i.e. the most accurate representation of the systematic differences between catalogues. Expansion in spherical functions (Brosche's method) gives less accurate representation of the systematic differences. One can see that the first two methods do not adequately reflect the actual errors of the modern RSCs. As to the first two methods, they seem to be not adequate to the actual errors of the modern RSCs.
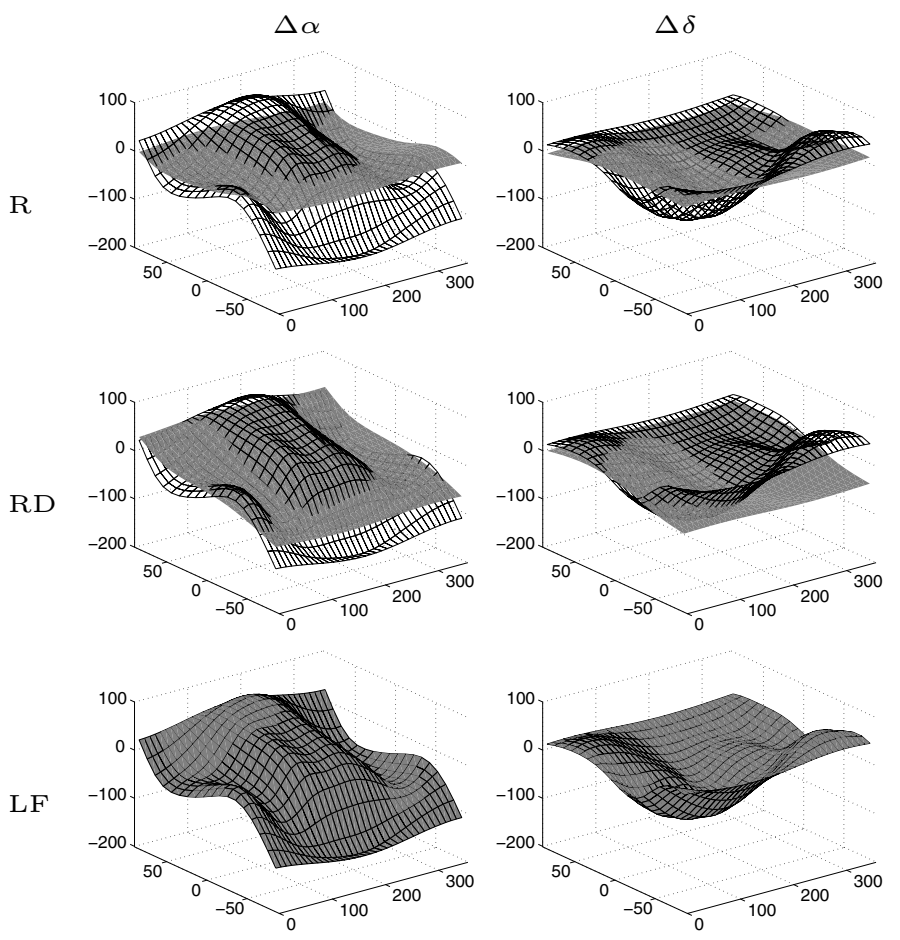

Fig. 3. An example of the quality of analytical representation of the differences between the input catalogues and the ICRF with different methods using the USNO catalogue. From top to bottom: original differences (gridded surface) and the result of approximation using the rotation model, the same for rotation with deformation, the same for Legendre-Fourier functions. Horizontal axes show right ascension (right) and declination (left) in degrees. Unit: $\mu$ as.

Table 2. WRMS differences between the input catalogues and the ICRF before (Raw) and after approximation of the systematic differences (see notation of the methods in text). Results related to the LF method, providing the best approximation, are shown in bold. Unit: $\mu$ as.

\begin{tabular}{lcccccccc}
\hline \hline & AUS & BKG & DGFI & JPL & USNO & GSFC & MAO & SHAO \\
\hline Raw & 304 & 277 & 343 & 331 & 253 & 277 & 294 & 273 \\
R & 301 & 271 & 342 & 308 & 249 & 274 & 286 & 271 \\
RD & 299 & 265 & 342 & 308 & 247 & 273 & 285 & 270 \\
B & 170 & 177 & 237 & 238 & 172 & 191 & 203 & 193 \\
LF & $\mathbf{1 0 6}$ & $\mathbf{1 2 5}$ & $\mathbf{1 6 4}$ & $\mathbf{1 7 2}$ & $\mathbf{1 2 2}$ & $\mathbf{1 4 4}$ & $\mathbf{1 5 2}$ & $\mathbf{1 4 5}$ \\
& & & & & $\Delta \delta$ & & & \\
Raw & 337 & 225 & 284 & 277 & 221 & 238 & 251 & 240 \\
R & 337 & 225 & 284 & 273 & 221 & 238 & 251 & 240 \\
RD & 333 & 224 & 283 & 273 & 221 & 237 & 251 & 239 \\
B & 180 & 159 & 178 & 182 & 152 & 158 & 169 & 166 \\
LF & $\mathbf{1 1 1}$ & $\mathbf{1 0 9}$ & $\mathbf{1 1 2}$ & $\mathbf{1 2 7}$ & $\mathbf{1 0 4}$ & $\mathbf{1 0 6}$ & $\mathbf{1 3 4}$ & $\mathbf{1 1 1}$ \\
\hline
\end{tabular}

\section{Combined catalogue in the ICRF system}

In the next step, the systematic differences between the input catalogues and the ICRF found by the LF method, as described in the previous section, were applied to all of the input catalogues with the intention of transforming them to the ICRF system. To provide further procedures for construction of combined catalogues, the same number (namely 36) of terms of the expansion (7) was used for all the input catalogues. Subsequently, the coordinates of all the sources in the transformed catalogues were averaged with weights depending on the formal errors of coordinates. Based on this, the combined catalogue RSC(PUL)07C01 was constructed. This catalogue, containing all the 968 sources 


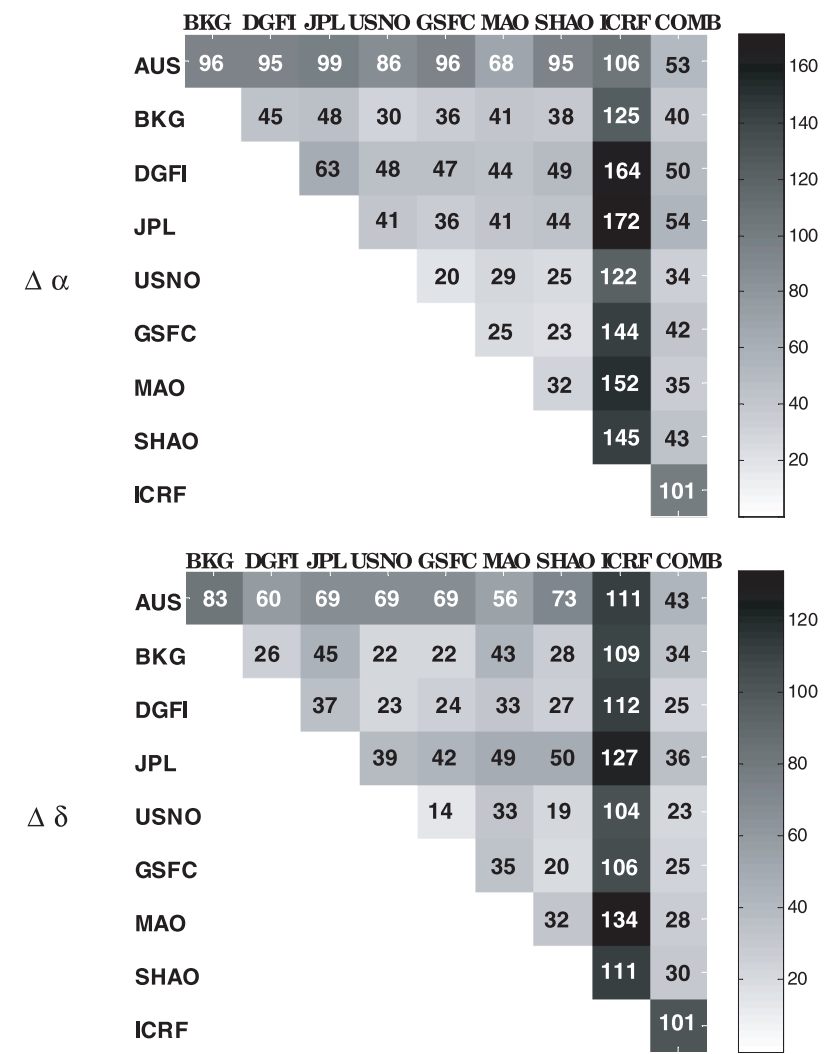

Fig. 4. WRMS differences between the input catalogues transformed to the ICRF system and the combined catalogue RSC(PUL)07C01. Unit: $\mu$ as.
$\Delta \alpha$

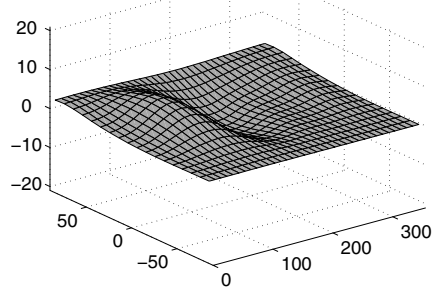

$\Delta \delta$

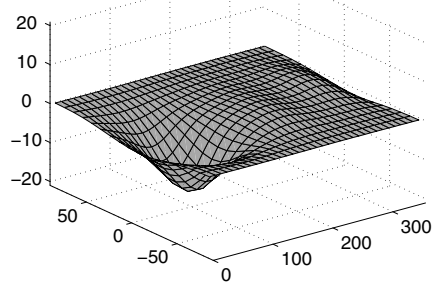

Fig. 5. Differences between RSC(PUL)07C01 and the ICRF. Horizontal axes show right ascension (right) and declination (left) in degrees. Unit: $\mu$ as.

present in the input catalogues, can be considered as a stochastic improvement of the ICRF. Figure 4 shows the WRMS differences between the input catalogues transformed to the ICRF system and RSC(PUL)07C01.

From Fig. 4, one can conclude that the differences between the input catalogues contain not only the systematic part described by analytical representation, but also significant stochastic components.

Figure 5 shows the systematic difference between combined catalogue RSC(PUL)07C01 and the ICRF. One can see that the catalogue RSC(PUL)07C01 represents the ICRF system at a level of about $10 \mu$ as.

\section{Final combined catalogue}

The final combined catalogue was constructed in the following way. For each input catalogue, we have a set of coefficients $b$ of expansion (7) of the systematic differences between a given
Table 3. Weights of the input catalogues applied during combination, averaged over the sky.

\begin{tabular}{ccccccccc}
\hline \hline & AUS & BKG & DGFI & GSFC & JPL & MAO & SHAO & USNO \\
\hline$\alpha$ & 0.246 & 0.671 & 0.464 & 1.993 & 0.254 & 0.558 & 1.792 & 2.062 \\
$\delta$ & 0.205 & 1.220 & 0.586 & 1.921 & 0.446 & 0.541 & 1.459 & 1.927 \\
\hline
\end{tabular}

Table 4. WRMS differences between the input catalogues and the combined catalogue RSC(PUL)07C02. Unit: $\mu$ as.

\begin{tabular}{lcccccccc}
\hline \hline & AUS & BKG & DGFI & GSFC & JPL & MAO & SHAO & USNO \\
\hline$\alpha$ & 183 & 89 & 50 & 49 & 118 & 57 & 50 & 45 \\
$\delta$ & 165 & 57 & 49 & 49 & 76 & 55 & 39 & 36 \\
\hline
\end{tabular}
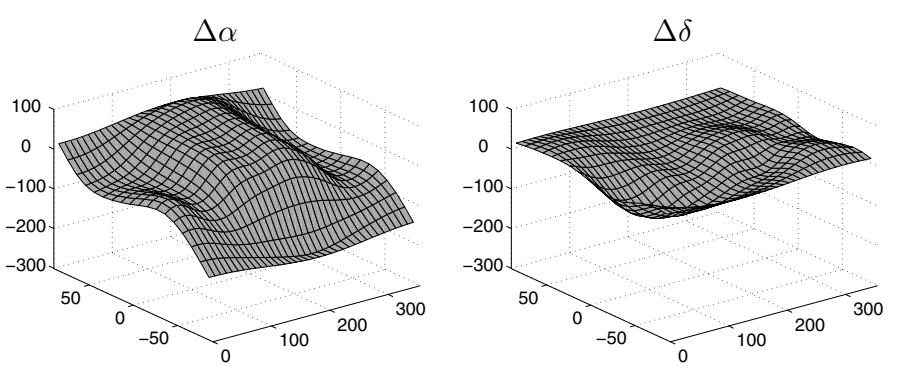

Fig. 6. Differences between RSC(PUL)07C02 and the ICRF. Horizontal axes show right ascension (right) and declination (left) in degrees. Unit: $\mu$ as.

catalogue and the ICRF in the Legendre-Fourier functions. Let us call this set of coefficients the "system" of a given catalogue, or individual system. Thus we have got eight systems corresponding to the eight input catalogues.

At the first iteration, the individual systems were averaged without weights, and thus the first approximation of the average system was obtained. The WRMS differences between this average system and the systems of the input catalogues for the bins $10^{\circ}(\alpha) \times 5^{\circ}(\delta)$ were then computed. These WRMS values were used for weighting of the individual systems at the second iteration. The final average system was then computed as a weighted average of the individual systems. Although we used different weights of the input catalogues for different bins, they are close for all the bins in each catalogue. Final weights of the catalogues averaged over the sky are given in Table 3 .

The derived final average system, in fact, the weighted average of coefficients $b$ of expansion (7), was used to compute the corrections to coordinates of all 968 radio sources of the RSC(PUL)07C01 catalogue in accordance with (7), and these corrections were added to the RSC(PUL)07C01 positions. As a result, the final combined catalogue, RSC(PUL)07C02 has been obtained. It can be considered as both a stochastic and a systematic improvement of the ICRF. In Table 4, WRMS differences between the input catalogues and the combined catalogue RSC(PUL)07C02 are shown.

Comparison of RSC(PUL)07C02 and the ICRF is presented in Fig. 6, which shows the result of expansion of the differences between the two catalogues in Legendre-Fourier functions. Results of this comparison lead us to the supposition that ICRF may have significant systematic errors.

\section{Comparison with observations}

It is important to assess the accuracy of the obtained catalogue (as well as other CRF realizations). Unfortunately, existing 
Table 5. Scatter of the celestial pole offset time series obtained with two catalogues. The FCN column shows the scatter with respect to the FCN model, the WADEV column shows the weighted Allan deviation. Unit: $\mu$ as.

\begin{tabular}{lrrrrrc}
\hline \hline Catalogue & \multicolumn{3}{c}{ FCN } & \multicolumn{3}{c}{ WADEV } \\
& $X$ & \multicolumn{1}{c}{$Y$} & Mean & $X$ & $Y$ & Mean \\
\hline ICRF-Ext.2 & 103 & 101 & 102 & 113 & 109 & 111 \\
RSC(PUL)07C02 & 98 & 98 & 98 & 105 & 106 & 105 \\
\hline
\end{tabular}

methods of comparison of catalogues allow us to investigate only differences between catalogues. Here, we use a test that can help us to get an independent estimate of the quality of our combined catalogue. For this purpose, we compute two celestial pole offset time series from the processing of the R1 and R4 IVS observing programs, observed in the period 2002-2006 with two radio source catalogues, ICRF-Ext. 2 and RSC(PUL)07C02. We then compute two estimates of the scatter for these two time series. The first estimate is the WRMS difference between computed celestial pole offsets and the IAU2000A model supplemented with the Free Core Nutation contribution. The second estimate was computed as the weighted Allan deviation (WADEV) of the celestial pole offset time series. Details of this method of scatter estimation are given in Malkin (2007). The results of this test, presented in Table 5, show clear improvement of the scatter of celestial pole offset estimates when using combined catalogue.

\section{Conclusion}

In this paper, we have constructed a new combined catalogue of radio source coordinates. For this study we used eight catalogues submitted by IVS Analysis Centers in the framework of the IERS/IVS Pilot Project on the future realization of the ICRF.

We have examined four different methods of analytical representation of systematic differences between catalogues of radio source coordinates. Expansion of the systematic differences in Legendre-Fourier functions proved to be the most accurate method. Two methods usually used for comparison of the CRF realizations based on the positions of extragalactic radio sources catalogues, namely axes rotation and rotation with deformation (IERS method), seem to be unsuitable for investigation of the systematic differences of modern radio source catalogues, and evidently should be replaced by a more adequate comparison technique.

Two combined radio source catalogues have been constructed. The first of them, RSC(PUL)07C01, is obtained as a weighted average of the input catalogues, corrected for systematic differences with the ICRF given by the expansion in the Legendre-Fourier functions. It can be considered as a stochastic improvement of the current realization of ICRF. Analysis of the systematic differences between RSC(PUL)07C01 and the ICRF has shown that they are at a level of microarcseconds.

The final combined catalogue, RSC(PUL)07C02, was obtained from the first one, RSC(PUL)07C01, after applying weighted average systematic differences between the input catalogues and the ICRF. This catalogue provides both stochastic and systematic improvements of the ICRF.

To compare the RSC(PUL)07C02 catalogue with the ICRF, we used two tests based on estimation of the scatter of celestial pole offset time series, computed by processing of VLBI observations using the ICRF and the final combined catalogue. Both tests have shown less scatter of the series based on the combined catalogue.

The results obtained in this paper allow us to make the conclusion that ICRF-Ext.2 may have significant systematic errors, probably caused by fixing the coordinates of 212 defining sources to the values obtained in the first ICRF version of 1995.

Further development of this study may include:

- more detailed analysis of stochastic and systematic errors of radio source catalogues,

- estimation of the possible impact of the high-frequency systematic errors in source position on the orientation of the catalogue axes,

- analysis of the reasons for systematic differences between radio source catalogues,

- careful selection of the input catalogues and reference sources,

- elaboration of weighting method,

- development of more sensitive methods for the assessment of the accuracy of CRF realizations.

It would be also interesting to compare different methods of construction of combined radio source catalogues, in particular the so-called arc approach developed by Yatskiv \& Kur'yanova (1990).

Acknowledgements. The authors greatly appreciate all the IVS Analysis Centers providing their catalogues of the radio source positions used in this study. We thank the anonymous referee for careful reading of the manuscript and his helpful and constructive comments.

\section{References}

Arias, E. F., \& Bouquillon, S. 2004, A\&A, 422, 1105

Arias, E. F., Charlot, P., Feissel, M., \& Lestrade, J.-F. 1995, A\&A, 303, 604 Bien, R., Fricke, W., Lederle, T., \& Schwan, H. 1978, Veroffen. Astron. RechenInst., Heidelberg, No. 29

Brosche, P. 1966, Veroff. Astron. Rechen-Inst., Heidelberg, No. 17

Fey, A.L., Ma, C., Arias, E. F., et al. 2004, AJ, 127, 3587

Kur'yanova, A. N., \& Yatskiv, Ya. S. 1993, Kinemat. fiz. nebesnyh tel, 9, 15

Ma, C. 2001, in Proc. 15th Working Meeting on European VLBI for Geodesy and Astrometry, ed. D. Behrend, \& A. Rius, 187

Ma, C., Arias, E. F., Eubanks, T. M., et al. 1998, AJ, 116, 516

Malkin, Z. 2007, J. Geodesy, [doi: 10.1007/s00190-007-0181-x]

Schlueter, W., Himwich, E., Nothnagel, A., et al. 2002, Adv. Space Res., 30, 145

Walter, H. G. 1989a, A\&A, 210, 455

Walter, H. G. 1989b, A\&AS, 79, 283

Walter, H. G., \& Sovers, O. J. 2000, Astrometry of fundamental catalogues. The evolution from optical to radio reference frames (Berlin: Springer-Verlag)

Yatskiv, Ya. S., \& Kur'yanova, A. N. 1990, in: Inertial Coordinate System on the Sky, Proc. 141th IAU Symp., ed. J. H. Lieske, \& V. K. Abalakin, 295 